\title{
Phosphodiesterase 3 Inhibitors Suppress Oocyte Maturation and Consequent Pregnancy without Affecting Ovulation and Cyclicity in Rodents
}

\author{
A. Wiersma, ${ }^{\star}$ B. Hirsch, ${ }^{\ddagger}$ A. Tsafriri, ${ }^{\ddagger \S}$ R.G.J.M. Hanssen, ${ }^{\star}$ M. Van de Kant, ${ }^{\star}$ H.J. Kloosterboer, ${ }^{\star}$ M. Conti, ${ }^{\ddagger}$ and A.J.W. Hsueh ${ }^{\ddagger}$ \\ *Department of Pharmacology, N.V. Organon, P.O. Box 20, 5340 BH Oss, The Netherlands; ${ }^{\ddagger}$ Division of Reproductive Biology, \\ Department of Obstetrics and Gynecology, Stanford University School of Medicine, Stanford, California 94305-5317; and ${ }^{\S}$ Department of \\ Biological Regulation, Bernhard Zondek Hormone Research Laboratory, Weizmann Institute of Science, Rehovot 76100, Israël
}

\begin{abstract}
During each reproductive cycle, a preovulatory surge of gonadotropins induces meiotic maturation of the oocyte in the preovulatory follicle followed by ovulation. Although gonadotropins stimulate cAMP production in somatic cells of the follicle, a decrease in intra-oocyte cAMP levels is required for resumption of meiosis in oocytes. Based on the observed compartmentalization of the cAMP-degrading enzyme, phosphodiesterase, in follicular somatic and germ cells, inhibitors of phosphodiesterase 3 were used to block meiosis in ovulating oocytes in rodents. By this strategy, we demonstrated that fertilization and pregnancy could be prevented without disturbing follicle rupture and normal estrous cyclicity. In contrast to conventional contraceptive pills that disrupt ovarian steroidogenesis and reproductive cycles, the present strategy achieves effective contraception by selective blockage of oocyte maturation and development without alterations in ovulation and reproductive cyclicity. (J. Clin. Invest. 1998. 102:532-537.) Key words: dissociation oocyte maturation/ovulation - germinal-vesicle-stage oocytes • immature ovulated oocytes $\cdot \mathrm{PDE} \cdot$ contraception
\end{abstract}

\section{Introduction}

Mammalian oocyte development is characterized by prolonged meiotic arrest and gonadotropin-induced resumption of meiosis. During most of its growth phase, the oocyte is arrested at prophase I and is meiotically incompetent. In vitro and in vivo, oocytes acquire the ability to resume meiosis when oocytes approach their final size. In vivo, in healthy follicles meiosis is triggered by the preovulatory surge of luteinizing hormone $(\mathrm{LH})^{1}$ during each reproductive cycle. Resumption

Address correspondence to Anja Wiersma, Dept. of Pharmacology, RE 2106, NV Organon, 5340 BH Oss, The Netherlands. Phone: 31412-661645; FAX: 31-412-662542; E-mail: a.wiersma@organon.oss. akzonobel.nl

Received for publication 18 December 1997 and accepted in revised form 21 May 1998.

1. Abbreviations used in this paper: $\mathrm{CEO}$, cumulus-enclosed oocyte; DO, denuded oocyte; GVBD, germinal vesicle breakdown; hCG, human chorionic gonadotropin; IBMX, isobutyl methylxanthine; i.p., intraperitoneal; LH, luteinizing hormone; PDE, phosphodiesterase; PKA, protein kinase A; PMSG, pregnant mare serum gonadotropin; s.c., subcutaneous.

J. Clin. Invest.

(C) The American Society for Clinical Investigation, Inc. 0021-9738/98/08/0532/06 \$2.00

Volume 102, Number 3, August 1998, 532-537

http://www.jci.org of meiosis includes disintegration of the nuclear membrane, usually referred to as germinal vesicle breakdown (GVBD), chromatin condensation, chromosome segregation, and the extrusion of the first polar body shortly before follicle rupture (1). In most mammals, at ovulation the oocyte reaches metaphase II and is ready for fertilization by a spermatozoon. Oocytes that do not reach metaphase II cannot be fertilized due to inability of the spermatozoa to penetrate the oocyte plasma membrane and, if they penetrate, to form a sperm pronucleus (2-4).

The second messenger cAMP plays an important role in meiotic arrest of oocytes in both mammalian and amphibian species (5-8). A correlation between the meiotic status of an oocyte and its intracellular levels of cAMP was demonstrated, suggesting that elevated cAMP levels within the oocyte prevent its maturation (9-13). In mammalian oocytes, a decrease in intracellular levels of cAMP is believed to initiate meiotic resumption and probably triggers events that result in an irreversible commitment of the oocyte to undergo $\operatorname{GVBD}(12,14)$. It has been suggested that hypoxanthine, which is present in high concentration in follicular fluid and which maintains meiotic arrest of isolated oocytes (reviewed by Downs; 15), may be a natural blocker of oocyte maturation (16). The inhibitory action of hypoxanthine is mediated through the suppression of phosphodiesterase (PDE) activity (17). However, the possibility that other factors are involved cannot be ruled out (1).

The spontaneous maturation of rodent oocytes in vitro can be prevented by addition of PDE inhibitors or by derivatives of cAMP, both of which cause an increase in intra-oocyte cAMP levels $(8,18)$. PDEs, responsible for the breakdown of cAMP, consist of a large group of proteins with at least seven different families characterized (19-21). In Xenopus laevis oocytes, support for the role of PDE3 on oocyte maturation was already suggested $(22,23)$. Recently, it was reported that gonadotropin induction of oocyte maturation in rats involves compartmentalization and opposing changes in cAMP levels in follicular somatic and germ cells. PDE4 inhibitors that mainly act on the granulosa cells (i.e., increase cAMP levels in these cells) enhance LH induction of oocyte maturation in cultured follicles. In contrast, treatment with specific PDE3 inhibitors, which act directly on the oocyte, by increasing cAMP levels, suppresses the LH induction of GVBD in rat follicle-enclosed oocytes (24).

The dissociation of cAMP regulation by specific inhibitors of PDE subtypes differentially located in somatic and germ cell compartments of the follicle opens possibilities to develop new strategies for contraceptive intervention. In the present study, experiments were performed to demonstrate that treatment with PDE3 inhibitors suppress oocyte maturation and prevent fertilization in vivo in rodents. Due to their specific action on oocytes, normal ovulation and estrous cyclicity were maintained. 


\section{Methods}

Animals. All animals were kept in a temperature-controlled room $\left(20 \pm 2^{\circ} \mathrm{C}\right)$ with a $14-10$-h light-dark cycle (lights on from 06.00 to $20.00 \mathrm{~h}$ ). Food and water were available ad libitum.

For the studies involving mice, immature females (B6D2-F1, strain $\mathrm{C} 57 \mathrm{Bl} / 2 \mathrm{~J}$ ) obtained from Charles River (Sulzfeld, Germany) were housed in groups of six, together with a foster mother. For studies using immature rats, females (Sprague-Dawley, Indianapolis, IN) obtained from Simonson (Gilroy, CA) were housed in groups of three. For studies on adult rats, 7 -wk-old cyclic female virgin rats (strain Orga, derived from Wistar rats), obtained from Harlan (Zeist, The Netherlands) were housed in groups of six. These rats were screened for having a regular 4-d estrous cycle by taking vaginal smears (at $08.30 \mathrm{~h}$ in the morning), for $2 \mathrm{wk}$. Animal treatments were approved by the Animal Care Committees at Stanford and Organon.

Reagents. Oocytes were collected in Leibovitz's L-15 medium (GIBCO BRL, Paisley, UK), supplemented with $4 \mathrm{mM}$ hypoxanthine, $3 \mathrm{mg} / \mathrm{ml} \mathrm{BSA}, 0.23 \mathrm{mM}$ pyruvate, and $2 \mathrm{mM}$ glutamine. Isolated oocytes were cultured in $\alpha$-MEM (GIBCO BRL), supplemented with $3 \mathrm{mg} / \mathrm{ml}$ BSA, $0.23 \mathrm{mM}$ pyruvate, and $2 \mathrm{mM}$ glutamine. The PDE inhibitors, hypoxanthine (non-selective), milrinone (selective for PDE3), and zaprinast (selective for PDE5) were obtained from Sigma Chemical Co. (St. Louis, MO), rolipram (selective for PDE4) was from ICN (Costa Mesa, CA), whereas cilostamide (selective for PDE3) was a gift of H. Hidaka (Nagoya University, Nagoya, Japan). Isobutyl methylxanthine (IBMX) (non-selective), pimobendan (selective for PDE3), Org 9935 (selective for PDE3), and the two enantiomers of Org 9935, Org 20864 (-) and Org 20865 (+), as well as Humegon and Pregnyl, were obtained from NV Organon (Oss, The Netherlands). The PDE inhibitors were dissolved in stock solutions in DMSO (Sigma, Dieshofen, Germany), diluted to $0.1 \%$ DMSO, as indicated in the culture medium.

In vitro assays. Female mice (20-d-old) were injected subcutaneously (s.c.) with a single dose of human menopausal gonadotropin (Humegon) containing 20 IU follicle-stimulating hormone and 20 IU LH to stimulate multiple follicular development. $48 \mathrm{~h}$ after injection, the animals were killed by cervical dislocation. The antral follicles were punctured under a dissecting microscope using 30-gauge needles. Cumulus-enclosed oocytes (CEO) were collected and denuded oocytes (DO) were obtained by flushing CEO through a small, fine bore pipette. The oocytes were collected in fresh $\alpha$-MEM medium containing $4 \mathrm{mM}$ hypoxanthine and washed twice, before transfer to the test medium. The CEO and DO were cultured with or without different PDE inhibitors in 4-well multidish (Nunclon, Denmark) at $37^{\circ} \mathrm{C}$ and $100 \%$ humidity with $5 \% \mathrm{CO}_{2}$ in air. At the end of culture, the oocytes were examined for their meiotic status by an inverted microscope with Hoffman modulation contrast equipment (Nikon, Tokyo, Japan). Oocytes showing a clear nuclear membrane (germinal vesicle, GV) or an intact nucleolus were classified as meiotically arrested, whereas those showing no nuclear structures were classified as having undergone GVBD (25).

In vivo, oocyte maturation upon ovulation induction. Immature female mice $(20 \mathrm{~d})(n=24)$ were treated with 20 IU Humegon (s.c.) to stimulate multiple follicular development. 45 and $51 \mathrm{~h}$ later, mice were treated with vehicle, $(n=10,0.5 \%$ gelatin and $5 \%$ mannitol in water [gelatin/mannitol]) or Org $9935(500 \mathrm{mg} / \mathrm{kg}$ in gelatin $/ \mathrm{mannitol})$ by s.c. $(n=10)$ or intraperitoneal (i.p.) $(n=4)$ routes. To induce ovulation, 7.5 IU Pregnyl was administered s.c. $3 \mathrm{~h}$ after the first Org 9935 treatment. The following morning, mice were killed and oviducts were removed to isolate ovulated oocytes. The ovulated CEO were treated for $3 \mathrm{~min}$ with $0.3 \%$ of hyaluronidase (Sigma Chemical Co.) to remove the cumulus cells and to score for the presence of a germinal vesicle. Throughout isolation and examination, oocytes were bathed in L-15 medium supplemented with $10 \mu \mathrm{M}$ Org 9935 to prevent GVBD.

Immature female rats ( $26 \mathrm{~d}$ of age) were treated with $15 \mathrm{IU}$ pregnant mare serum gonadotropin (PMSG; Calbiochem, La Jolla, CA) to stimulate multiple follicular development. 48 and $53 \mathrm{~h}$ later, rats were treated with vehicle or PDE inhibitors (Cilostamide and Org 9935, suspended in gelatin/mannitol) orally (p.o.) in different concentrations. To induce ovulation, $10 \mathrm{IU}$ human chorionic gonadotropin (hCG) was administered s.c. $50 \mathrm{~h}$ after PMSG injection. $19 \mathrm{~h}$ later, ovulated oocytes were isolated from the oviduct and examined for their meiotic progression.

On the day of proestrus, adult rats with a regular cycle were treated orally (p.o.) with Org 9935 or vehicle twice at 11.00 and $17.00 \mathrm{~h}$. On estrous morning $(10.00 \mathrm{~h})$, the rats were killed and ovulated oocytes were scored for the presence of a germinal vesicle.

Monitoring of fertilization and pregnancy. 26-d-old immature female rats $(n=20)$ were treated with PMSG (15 IU), to stimulate multiple follicular development. At 48 and $53 \mathrm{~h}$ later, rats were treated at p.o. with Org 9935 (500 mg/kg in gelatin/mannitol). To induce ovulation, $10 \mathrm{IU}$ hCG was administered s.c. $50 \mathrm{~h}$ after PMSG injection, and a fertile male rat was introduced at $18.00 \mathrm{~h}$ on the same day to allow mating. Some animals were used to examine ovulated oocytes one day later (day 1), whereas others were used to examine blastocyst development $5 \mathrm{~d}$ after mating. In both groups, rats were killed at $8 \mathrm{am}$, and oviducts were removed to isolate ovulated oocytes or blastocysts.

For monitoring implantation and pregnancy, adult rats $(n=18)$ in proestrus were orally administered with Org $9935(200 \mathrm{mg} / \mathrm{kg}$ per injection) or vehicle at 09.00 and $17.00 \mathrm{~h}$ for $5 \mathrm{~d}$. A fertile male was introduced on proestrus evening and successful mating was confirmed by the presence of a copulation plug the next morning. To rule out non-specific effects of Org 9935 on implantation, Org 9935 treatment was delayed in some animals and started at estrous morning. At day 15 after mating, the number of live embryos was determined for all groups.

To monitor the effect of PDE3 inhibitor on estrous cycle regularity, rats having 4-d cycles were treated orally with Org $9935(200 \mathrm{mg} / \mathrm{kg})$ or vehicle twice a day (at 09.00 and $17.00 \mathrm{~h}$ starting on proestrus) for $13 \mathrm{~d}$. During the treatment period, cyclicity was monitored.

To examine the effect of PDE3 inhibition on cardiac function, six adult female rats were implanted with a heart rate transmitter (DATA SCIENCES International, St. Paul, MN) to allow registration of heart rate, gross behavioral activity, and body temperature in individual freely moving animals. $14 \mathrm{~d}$ after surgery, the rats were placed in separate cages and registration started after $3 \mathrm{~d}$ of adjustment in the registration room. Accordingly, rats in proestrus were treated orally with Org $9935(200 \mathrm{mg} / \mathrm{kg})$ or vehicle twice a day (at 09.00 and $17.00 \mathrm{~h}$ ) for $4 \mathrm{~d}$, and heart rate was registered every $10 \mathrm{~min}$ for $2 \mathrm{wk}$.

Statistics. For analysis of percentages of oocytes that underwent GVBD, the Mann-Whitney U test was used. The Student's $t$ test was used to evaluate all other data. The results are given as mean \pm SEM. A probability level of $P<0.05$ was taken as significant.

\section{Results}

Inhibition of oocyte maturation in vitro using subtype-specific $P D E$ inhibitors. To extend earlier studies that demonstrated the specificity of PDE3 inhibitors in preventing oocyte maturation in rats (24), we treated DO from preovulatory follicles of gonadotropin-treated mice. As shown in Table I, the spontaneous maturation of cultured oocytes was dose dependently blocked by the non-selective PDE inhibitors, hypoxanthine and IBMX, with an IC50 of 400 and $50 \mu \mathrm{M}$, respectively. Treatment with several PDE3 inhibitors, i.e., milrinone, pimobendan, cilostamide, and Org 9935, also effectively blocked oocyte maturation with $>100$-fold lower dose than the natural and non-selective inhibitor hypoxanthine (Table I). Differences in the IC50 values of the stereoisomers of Org 9935 (Org 20864 and Org 20865) confirmed the selectivity of PDE3 inhibition in oocytes. In contrast, a selective PDE4 inhibitor, roli- 
Table I. Suppression of Mouse Oocyte Maturation In Vitro by PDE Inhibitors

\begin{tabular}{llcc}
\hline Family of PDE inhibitors & Compound & $\begin{array}{c}\text { IC50 }(\mu \mathrm{M}) \\
\text { mouse } \\
\text { oocyte }\end{array}$ & $\begin{array}{c}\text { IC50 }(\mu \mathrm{M}) \\
\text { PDE3 in } \\
\text { cardiac cells }\end{array}$ \\
\hline Non-selective inhibitors & $\begin{array}{l}\text { Hypoxanthine } \\
\text { IBMX }\end{array}$ & 400 & \\
PDE3 inhibitors & Milrinone & 50 & 12 \\
& Pimobendan & 0.5 & 3.2 \\
& Cilostamide & 0.1 & 0.05 \\
& Org 9935 & 0.1 & 0.4 \\
& -Org 20864 $(-)$ & 0.01 & 0.1 \\
PDE4 inhibitor & Rolipram & 1.0 & 4.7 \\
PDE5 inhibitor & Zaprinast & 500 & $>100$ \\
& & 200 & 126 \\
\hline
\end{tabular}

Inhibitors for different families of the PDEs were used to evaluate their suppression of oocyte maturation. For comparison, IC50 values of these compounds to inhibit PDE3 in rabbit cardiac tissues are also shown (37, and/or Organon and Newhouse).

pram, and a PDE5 inhibitor, zaprinast, affected oocyte maturation only at concentrations $200-500$-fold higher than those PDE3 inhibitors, presumably due to non-specific actions. The relative potencies (IC50) of these compounds in cardiac preparations paralleled their effects on oocyte maturation, suggesting that in both cases the interaction with the catalytic site of PDE3 is responsible for the pharmacological effects (Table I).

Treatment with PDE3 inhibitors suppresses oocyte maturation without affecting follicle rupture. Immature mice were treated with gonadotropins to induce ovulation. Treatment with the PDE3 inhibitor (Org 9935), through s.c. or i.p. injections, did not interfere with the number of oocytes ovulated compared with controls (Fig. 1, top). Remarkably, the PDE3 inhibitor completely suppressed oocyte maturation (Fig. 1, bottom); i.p. administration resulted in a complete blockade of maturation of the ovulated oocytes $(0 \%$ GVBD in 262 oocytes), whereas s.c. administration caused a $90 \%$ blockade of oocyte maturation.

We further tested the dose-dependent action of Org 9935 and another PDE3 inhibitor, cilostamide, to suppress oocyte maturation during ovulation in immature rats. The two PDE3 inhibitors decreased oocyte maturation in a dose-dependent manner without significantly affecting the number of ovulated oocytes (Fig. 2). Cilostamide was three times more potent than Org 9935 in inhibiting oocyte maturation. In Fig. 3 (top), a representative ovulated oocyte removed from a PDE3 inhibitortreated rat is compared with those from a control animal. The presence of the germinal vesicle in the treated animal is evident (Fig. 3, top left), whereas a polar body is visible in the control animal (Fig. 3, top right).

PDE3 inhibitors block fertilization and pregnancy. On day 1 after mating, none of the ovulated oocytes in immature rats receiving Org 9935 showed signs of fertilization (Table II). Likewise, no blastocyst was found at day 5 after mating. In contrast, matured oocytes and blastocysts were routinely found in controls. In Fig. 3 (lower left), a representative photograph of an

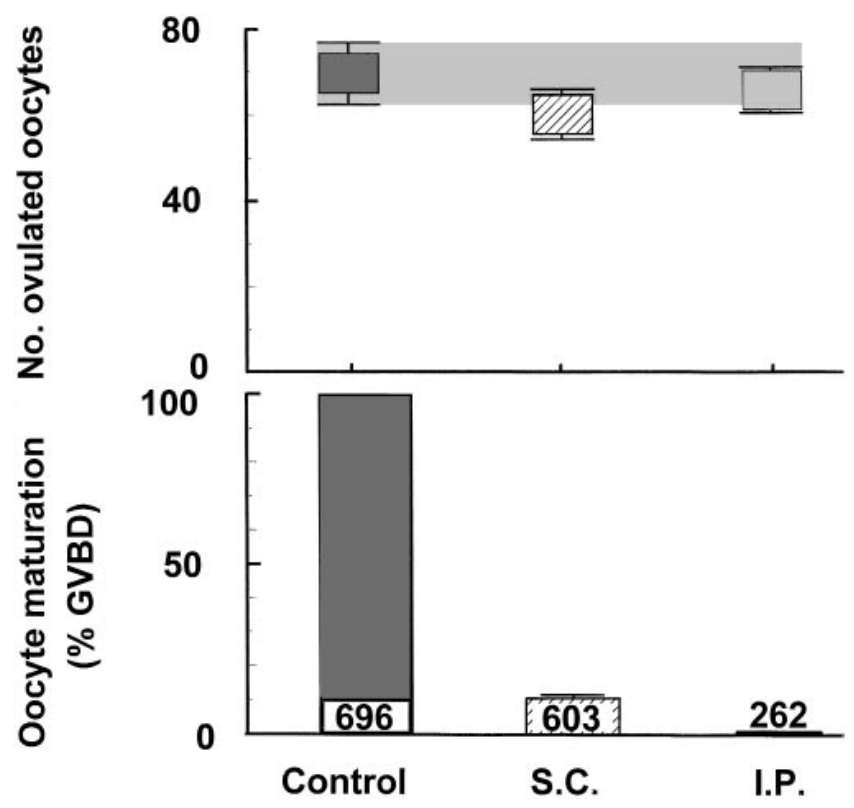

Figure 1. The effect of in vivo treatment with the PDE3 inhibitor, Org 9935, on ovulation and oocyte maturation in immature mice. Top: number of ovulated oocytes in the oviducts of control, subcutaneous (s.c.) and intraperitoneal (i.p.)-treated immature mice. Bottom: the stage of maturation of ovulated oocytes in different groups. Mean \pm SEM and the number of oocytes examined are shown.

ovulated immature oocyte surrounded by associated spermatozoa is shown (day 1 of fertilization). The GV and the nucleolus are clearly visible while the sperm head is not fused with the immature oocyte. Most of the GV-stage oocytes were not penetrated by spermatozoa. In the few that were penetrated, no pronucleus was observed. In Fig. 3 (lower right), an ovulated mature oocyte from the control group fertilized by a sperm is shown.

As shown in Table II, treatment with Org 9935 in adult cyclic rats completely blocked viable pregnancy. Compared with the mean of 14.2 live fetuses on day 15 of pregnancy in vehicletreated rats, treatment with Org 9935, starting at proestrus, led to only one viable embryo in one of the six treated rats. In contrast, delayed administration of the PDE3 inhibitor, starting on the morning after mating, did not affect the number of living embryos, indicating that the pharmacological treatment does not have non-specific toxic effects on the embryo.

Although treatment with PDE3 inhibitors did not affect follicle rupture, one cannot rule out the possibility that ovarian hormonal secretion is affected. We further monitored estrous cycle pattern in treated animals and found no adverse effects on the estrous cycle. Just like vehicle-treated females, 10 out of 10 females kept their regular 4-d-cycle after oral administration of Org 9935 for $13 \mathrm{~d}$.

Because the present PDE3 inhibitors originally have been developed for cardiovascular modulation, heart rate in treated rats was recorded as well. Administration of Org 9935 for $5 \mathrm{~d}$ resulted in an overall increase in heart rate (by $73.75 \pm 5.66$ beats per min). Furthermore, no apparent behavioral abnormality was observed. 


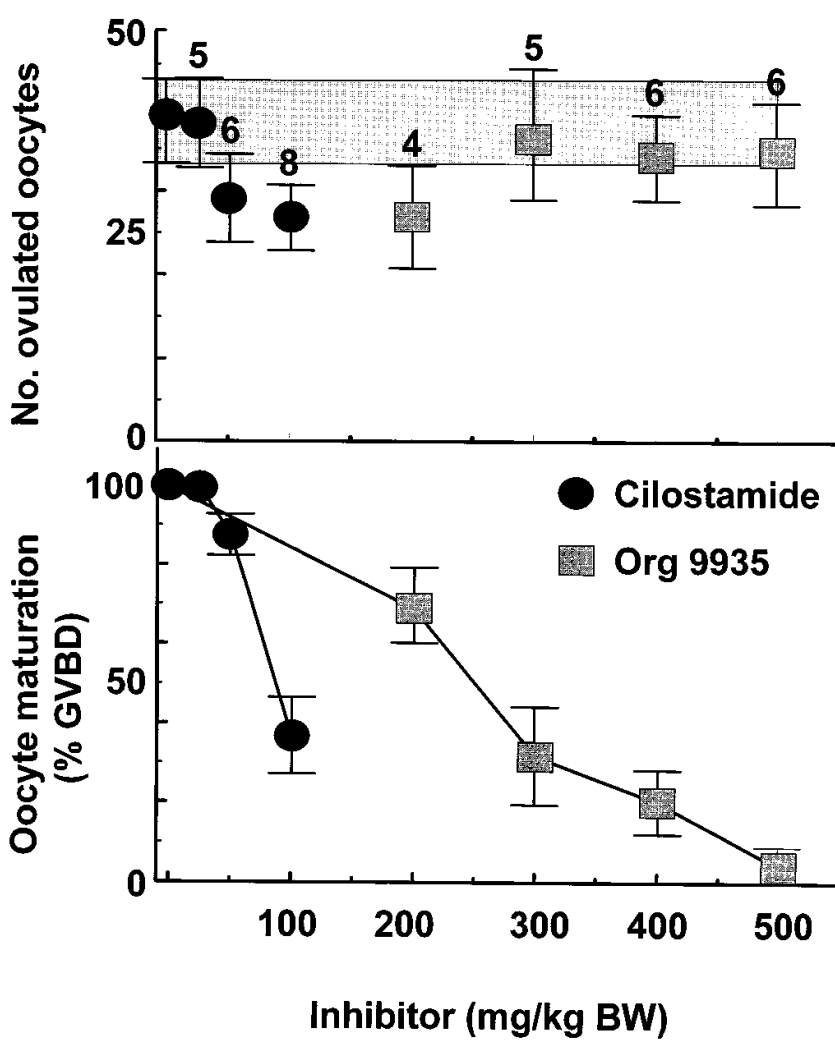

Figure 2. The in vivo effects of treatments with two PDE3 inhibitors, cilostamide and Org 9935, on ovulation and oocyte maturation in immature rats. Top: number of ovulated oocytes in the oviducts of control, cilostamide-treated (p.o.) and Org 9935-treated (p.o.) immature rats. The number of animals tested for each concentration of PDE3 inhibitor is given. Bottom: the maturation status of ovulated oocytes in different groups. Mean \pm SEM are given.

\section{Discussion}

One of the most widely used methods of contraception is the oral contraceptive hormone pill known to disrupt the pattern of gonadotropin secretion and ovulation. Although of undisputed efficacy, the long term exposure to estrogen/progestogen that this method involves is incidentally associated with side effects. Here we describe a novel approach to the control of fertility in the female, based on the prevention of oocyte maturation at the time of ovulation using inhibitors of enzymes involved in cAMP signal transduction. After treatment by PDE3 inhibitors, immature oocytes that are unable to be fertilized were ovulated without affecting ovarian function and the reproductive cycle.

In the intact follicle, a balance is maintained between meiotic arrest and meiotic induction in fully-grown oocytes $(1,15)$. Taking advantage of the compartmentalization of various PDE subtypes in the follicle (24), we were able to manipulate the cAMP-dependent pathway in the oocyte without affecting cAMP-mediated functions in the granulosa cells in rodents. All the data collected indicate that a rise in cAMP blocks meiotic resumption not only in vitro, but also in vivo. An increase in intra-oocyte cAMP levels results in an increase in protein kinase A (PKA) activation, and subsequent phosphorylation of specific proteins suggesting that the use of a PKA agonist may also allow meiotic arrest in vivo (26). Conversely, precocious oocyte maturation in hamsters was found after treatment with PKA inhibitor (27). Because the present PDE3 inhibitors does not affect PDE4 found in granulosa cells of ovarian follicles, steroidogenesis by somatic follicle cells appears not to be affected, neither was the reproductive cycle disturbed.

The complete dissociation of oocyte maturation and ovulation in cycling rats, resulting in the ovulation of immature oocytes not capable of being fertilized, has not been reported previously. In naturally ovulating mice, rats, and pigs, oocyte maturation and follicular rupture at ovulation are separated only rarely (1). One study reveals that precocious treatment of pigs with hCG resulted in the release of immature ova from immature follicles (28).

A crucial role for PDEs in oocyte maturation is already indicated by the phenotype caused by mutation in a Drosophila melanogaster dnc locus. The female germ line homozygous for a dunce null mutation produces oocytes that fail to develop (29). Furthermore, in X. laevis oocytes, inhibitors of PDE3 were able to block the insulin-induced oocyte maturation (22, 23). With a pharmacological ablation strategy, we showed that blockade of PDE3 activity causes an arrest in oocyte maturation and may allow control of fertility in rodents. These findings reinforce the conclusion that the activity of a PDE is indispensable for oocyte maturation in various species studied.

The rank of potency of the different compounds in blocking oocyte maturation in the mouse in vitro closely follows the potency for inhibition of PDE3 in cardiac cells. Inhibitors of

Table II. Effects of Org 9935 on Fertilization and Pregnancy in Rats

\begin{tabular}{|c|c|c|c|}
\hline & $\begin{array}{c}\text { Day } 1 \\
\text { Fertilized ova/rat }\end{array}$ & $\begin{array}{c}\text { Day } 5 \\
\text { Blastocysts/rat }\end{array}$ & $\begin{array}{c}\text { Day } 15 \\
\text { Viable embryos }\end{array}$ \\
\hline \multicolumn{4}{|c|}{ Super-ovulated, immature rats } \\
\hline Control & $\begin{array}{c}24.2 \pm 14.8 \\
(n=5)\end{array}$ & $\begin{array}{c}11.6 \pm 2.9 \\
(n=5)\end{array}$ & \\
\hline $\begin{array}{l}\text { Treatment } \\
500 \mathrm{mg} / \mathrm{kg}\end{array}$ & $\begin{array}{c}0 \\
(n=4)\end{array}$ & $\begin{array}{c}0 \\
(n=6)\end{array}$ & \\
\hline \multicolumn{4}{|l|}{ Cycling rats } \\
\hline Control & & & $\begin{array}{c}14.2 \pm 1.0 \\
(n=6)\end{array}$ \\
\hline $\begin{array}{l}\text { Treatment } \\
200 \mathrm{mg} / \mathrm{kg} \text { (day 0-4) }\end{array}$ & & & $\begin{array}{c}* \\
(n=6)\end{array}$ \\
\hline $\begin{array}{l}\text { Delayed treatment } \\
200 \mathrm{mg} / \mathrm{kg} \text { (day } 1-4)\end{array}$ & & & $\begin{array}{c}13.5 \pm 0.8 \\
(n=6)\end{array}$ \\
\hline
\end{tabular}

*One rat had only one embryo. Superovulated immature rats were treated on day 0 with $500 \mathrm{mg} / \mathrm{kg}$ Org 9935 or vehicle (gelatin/mannitol). Proestrus rats were mated with fertile males, as confirmed by the presence of a vaginal plug. Fertilized ova in the ampulla and blastocysts from the uterus were monitored on day 1 and 5, respectively. In addition, adult cyclic female rats were treated for $5 \mathrm{~d}$ either with vehicle or with Org $9935(200 \mathrm{mg} / \mathrm{kg})$, and pregnancy outcome monitored based on viable embryos on day 15 . A third group, with delayed treatment, received vehicle on day 0 , but Org 9935 treatment $(200 \mathrm{mg} / \mathrm{kg})$ from day 1 to 4 , to examine the effects of the treatment on implantation. Mean \pm SEM are given. 


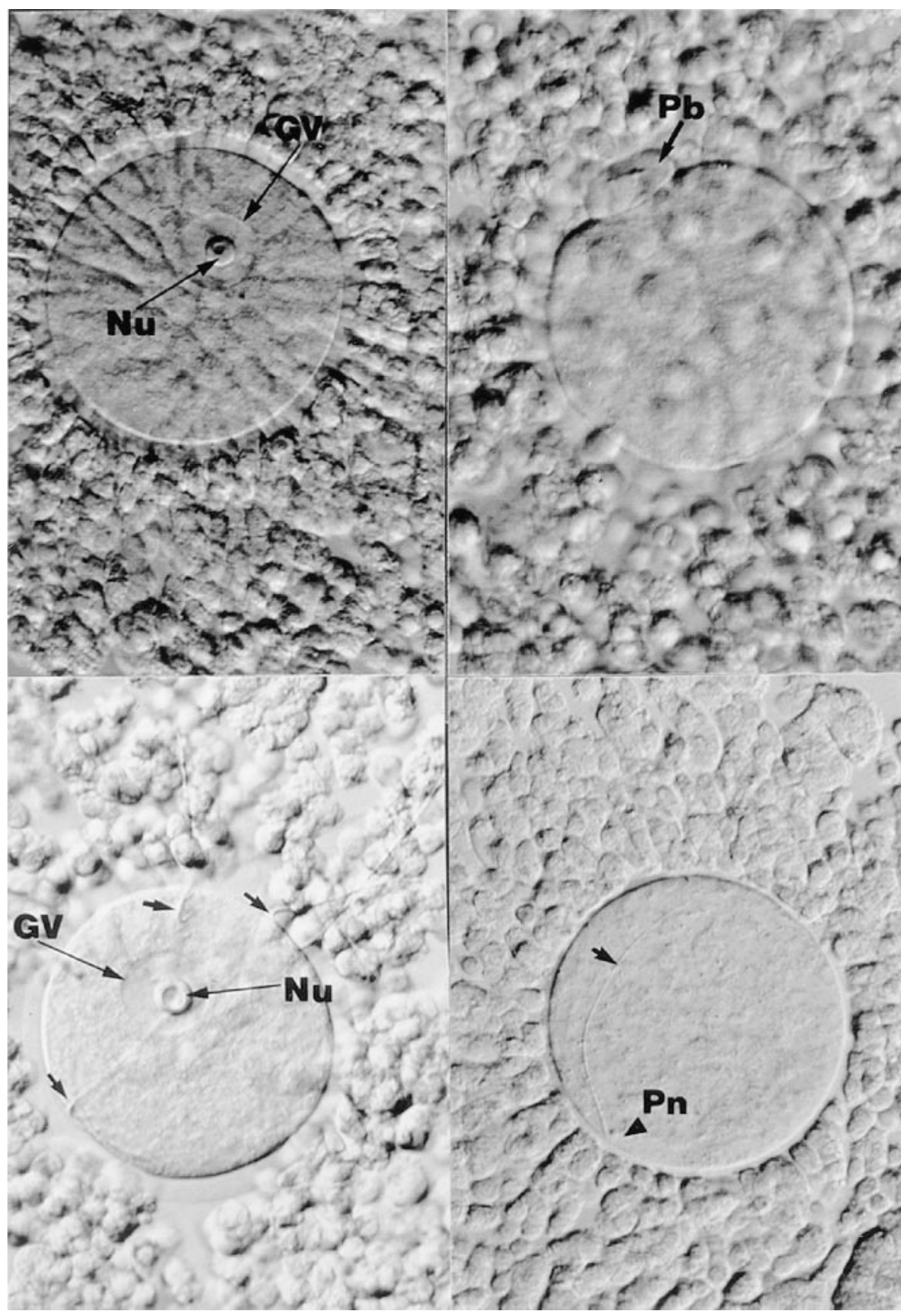

Figure 3. The effect of treatment with PDE3 inhibitor, Org 9935, on oocyte maturation and subsequent fertilization. Representative pictures of Normasky interference microscopy on oviductal oocytes isolated on the morning of estrus are presented. Top: oocytes from unmated animals. On the left, immature oocyte from a rat treated with the PDE3 inhibitor showing intact nuclear membrane, germinal vesicle $(G V)$, and nucleolus $(N u)$; On the right, a mature ovum from a vehicletreated control rat showing a polar body $(P b)$. Bottom: oocytes from mated rats. On the left, immature oocyte from a PDE3 inhibitor-treated rat showing several sperm heads (arrows) within the perivitelline space. On the right, a fertilized ovum from a vehicle-treated control rat with pronucleus $(P n)$ and sperm tail in the perivitelline space (arrow). In all cases, the cumulus cells underwent expansion and mucification.
PDEs other than PDE3 were invariably poor inhibitors of oocyte maturation, and non-selective PDE inhibitors were less potent than PDE3 inhibitors. The stereoselectivity of the two isomers of Org 9935 was also found in the inhibition of oocyte maturation. Collectively, these findings support the conclusion that blockade of oocyte maturation is the result of the interaction of the different drugs with the catalytic site of PDE3 present in the oocyte. The finding that PDE3 mRNA expression is detected by in situ hybridization in rat oocytes further strengthens this conclusion $(24,30)$. The in vitro observation on oocyte maturation can be extended to the in vivo situation because two specific PDE3 inhibitors suppressed oocyte maturation in vivo as well.

Type 3 PDEs play important roles in processes other than oocyte maturation including hormonal regulation of lipolysis, platelet aggregation, myocardial contractility, and smooth muscle relaxation. There are two different gene products for PDE3, one predominantly expressed in adipocytes and one in cardiac tissue. The adipocytes PDE3 subtype (PDE3B) is involved in hormonal regulation of lipolysis and glycogenolysis, whereas regulation of myocardial and smooth muscle contractility appears to be the function of the PDE3A subtype. The observation that the cardiac-type, PDE3A, mRNA is expressed at high levels in the rat oocytes suggests a new function for this gene product $(24,30)$. Indeed, treatment with Org 9935 blocked oocyte maturation as well as it induced an increase in heart rate. As only compounds that inhibit the oocyte PDE3A without affecting PDE3A expressed in the cardiovascular system can be considered for contraceptive purposes, the exact structure of the PDE3A expressed in the mammalian oocyte should be studied to determine if it is different from the PDE3 expressed in somatic cells. It has been reported that an alternative promoter in the PDE3A gene generates unique transcripts and proteins in the placenta (31). Given the extensive alternative splicing found for most of the PDE genes (19-21, 32-35), the oocyte PDE3A may also have unique properties, thus, al- 
lowing the design of a contraceptive strategy devoid of unwanted side effects.

Because contraceptive drugs are used by healthy individuals over a long period of time (36), future studies should aim towards the development of drugs that target only one single cell type (the oocyte in the preovulatory follicle) during each menstrual cycle. The present strategy of oocyte-specific PDE inhibition, when improved, could allow effective contraception by selective blockage of oocyte function without alterations in normal ovulation and reproductive cyclicity.

\section{Acknowledgments}

The authors wish to thank A. Tsafriri (Weizmann Institute of Science, Rehovot, Israël) for technical assistance and M. Shahid (N.V. Organon, Newhouse, Scotland) for valuable discussions. Cilostamide was a generous gift of H. Hidaka (Nagoya University, Nagoya, Japan).

The work described was in part supported by National Institutes of Health P50 HD31398 grant and by a grant from Organon (both to M. Conti).

\section{References}

1. Tsafriri, A., and N. Dekel. 1994. Molecular mechanisms in ovulation. In Molecular Biology of Female Reproductive System. J.K. Findlay, editor. Academic Press, San Diego, CA. 207-258.

2. Iwamatsu, T., and M.C. Chang. 1972. Sperm penetration in vitro of mouse oocytes at various times during maturation. J. Reprod. Fertil. 31:237-247.

3. Nima, K., and M.C. Chang. 1975. Fertilization of rat eggs in vitro at various times before and after ovulation with special reference to fertilization of ovarian oocytes matured in culture. J. Reprod. Fertil. 43:435-451.

4. Usui, N. 1996. Morphological differences in nuclear materials released from hamster sperm heads at an early stage of incorporation into immature oocytes, mature oocytes, or fertilized eggs. Mol. Reprod. Dev. 44:132-140.

5. Tsafriri, A., H.R. Lindner, U. Zor, and S.A. Lamprecht. 1972. In vitro induction of meiotic division in follicle-enclosed rat oocytes by LH, cyclic AMP and prostaglandin E2. J. Reprod. Fertil. 31:39-50.

6. Magnusson, C., T. Hillensjo, A. Tsafriri, R. Hultborn, K. Ahren, and W.J. Lemaire. 1977. Oxygen consumption of maturing rat oocytes. Biol. Reprod. 17: 9-15.

7. Eppig, J.J. 1989. The participation of cyclic adenosine monophosphate (cAMP) in the regulation of meiotic maturation of oocytes in the laboratory mouse. J. Reprod. Fertil. 38:3-8.

8. Downs, S.M., S.A.J. Daniel, E.A. Bornslaeger, P.C. Hoppe, and J.J. Eppig. 1989. Maintenance of meiotic arrest in mouse oocytes by purines: modulation of cAMP levels and cAMP phosphodiesterase activity. Gamete Res. 23: 323-334.

9. Bornslaeger, E.A., M.W. Wilde, and R.M. Schultz. 1984. Regulation of mouse oocyte maturation: involvement of cyclic AMP phosphodiesterase and calmodulin. Dev. Biol. 105:488-499.

10. Dekel, N., T.S. Lawrence, N.B. Gilula, and W.H. Beers. 1981. Modulation of cell-to-cell communication in the cumulus-oocyte complex: morphology and ovulatory changes. Dev. Biol. 86:356-362.

11. Aberdam, E., E. Hanski, and N. Dekel. 1987. Maintenance of meiotic arrest in isolated rat oocytes by the invasive adenylate cyclase of Bordetella pertussis. Biol. Reprod. 36:530-535.

12. Schultz, R.M., R.R. Montgomery, and J.R. Belanoff. 1983. Regulation of mouse oocyte meiotic maturation: implication of a decrease in oocyte cAMP and protein dephosphorylation in commitment to resume meiosis. Dev. Biol. 97:264-273.

13. Salustri, A., S. Petrungaro, M. DeFelici, and G. Siracusa. 1985. Effect of follicle-stimulating hormone on cyclic adenosine monophosphate level and on meiotic maturation in mouse cumulus cell enclosed oocytes cultured in vivo. Biol. Reprod. 33:797-802.
14. Vivarelli, E., M. Conti, M. DeFelici, and G. Siracusa. 1983. Meiotic resumption and intracellular cAMP levels in mouse oocytes treated with compounds which act on cAMP metabolism. Cell Differ. 12:271-276.

15. Downs, S.M. 1995. Ovulation 2: control of the resumption of meiotic maturation in mammalian oocytes. In Gametes-The Oocyte. J.D. Grudzinskas and J.L. Yovich, editors. Cambridge University Press, Cambridge, UK. 150-192.

16. Downs, S.M., D.L. Coleman, P.F. Ward-Bailey, and J.J. Eppig. 1985. Hypoxanthine is the principal inhibitor of murine oocyte maturation in a low molecular weight fraction of porcine follicular fluid. Proc. Natl. Acad. Sci. USA. 82:454-458.

17. Downs, S.M. 1993. Purine control of mouse oocyte maturation: evidence that nonmetabolized hypoxanthine maintains meiotic arrest. Mol. Reprod. Dev. 35: 82-94.

18. Cho, W.K., S. Stern, and J.D. Biggers. 1974. Inhibitory effect of dibutyryl cAMP on mouse oocyte maturation in vitro. J. Exp. Zool. 187:383-386.

19. Conti, M., G. Nemoz, C. Sette, and E. Vicini. 1995. Recent progress in understanding the hormonal regulation of phosphodiesterases. Endocr. Rev. 16 : 370-389.

20. Beavo, J.A. 1995. Cyclic nucleotide phosphodiesterases: functional implications of multiple isoforms. Physiol. Rev. 75:725-748.

21. Manganiello, V.C., M. Taira, E. Degerman, and P. Belfrage. 1995. Type III cGMP-inhibited cyclic nucleotide phosphodiesterases (PDE 3 gene family). Cell. Signalling. 7:445-455.

22. Sadler, S.E. 1991. Type III phosphodiesterase plays a necessary role in the growth-promoting actions of insulin, insulin-like growth factor-I, and $\mathrm{Ha}$ p21ras in Xenopus laevis oocytes. Mol. Endocrinol. 5:1939-1947.

23. Sadler, S.E. 1991. Inhibitors of phosphodiesterase III block stimulation of Xenopus laevis oocyte ribosomal S6 kinase activity by insulin-like growth factor-I. Mol. Endocrinol. 5:1947-1954.

24. Tsafriri, A., S.Y. Chun, R. Zhang, A.J.W. Hsueh, and M. Conti. 1996. Oocyte maturation involves compartmentalization and opposing changes of cAMP levels in follicular somatic and germ cells-studies using selective phosphodiesterase inhibitors. Dev. Biol. 178:393-402.

25. Tsafriri, A., and P.F. Kraicer. 1972. The time sequence of ovum maturation in the rat. J. Reprod. Fertil. 31:39-50.

26. Schultz, R.M. 1986. Molecular aspects of mammalian oocyte growth and maturation. In Experimental Approaches to Mammalian Embryonic Development. J. Rosant and R.A. Pederson, editors. Cambridge University Press, Cambridge, UK. 195-237.

27. Rosehellekant, T.A., and B.D. Bavister. 1996. Precocious oocyte maturation is induced by an inhibitor of cAMP-dependent protein kinase in the intact golden hamster. Mol. Reprod. Dev. 44:250-255.

28. Hunter, R.H., B. Cook, and T.G. Baker. 1976. Dissociation of response to injected gonadotropin between the Graafian follicle and oocyte in pigs. $\mathrm{Na}$ ture. 260:156-158.

29. Bellen, H.J., B.K. Gregory, C.L. Olsson, and J.A. Kiger. 1987. Two Drosophila learning mutants, dunce and rutabaga, provide evidence of a maternal role of cAMP embryogenesis. Dev. Biol. 121:432-444.

30. Rheinhardt, R.R., E. Chin, J. Zhou, M. Taira, T. Murata, and V.C. Manganiello. 1995. Distinctive anatomical patterns of gene expression for cGMPinhibited cyclic nucleotide phosphodiesterases. J. Clin. Invest. 95:1528-1538.

31. Kasuya, J., H. Goko, and Y. Fujita-Yamaguchi. 1995. Multiple transcripts for the human cardiac form of the cGMP-inhibited cAMP phosphodiesterase. J. Biol. Chem. 270:14305-14312.

32. Manganiello, V.C., T. Murata, M. Taira, P. Belfrage, and E. Degerman 1995. Diversity in cyclic nucleotide phoshphodiesterase isoenzyme families. Arch. Biochem. Biophys. 322:1-13.

33. Nicholson, C.D., R.A.J. Chaliss, and M. Shahid. 1991. Differential modulation of tissue function and therapeutic potential of selective inhibitors of cyclic nucleotide phosphodiesterase isoenzymes. Trends Pharmacol. Sci. 12:19-27.

34. Nicholson, C.D., M. Shahid, J. Bruin, E. Barron, I. Spiers, J. DeBoer, R.G.M. VanAmsterdam, J. Zaagsma, J.J. Kelly, M.A. Giembycz, and P.J. Barnes. 1995. Characterization of ORG 20241, a combined phosphodiesterase IV/III nucleotide phosphodiesterase inhibitor for asthma. J. Pharmacol. Exp. Ther. 274:678-687.

35. Thompson, W.J. 1991. Cyclic nucleotide phosphodiesterases: pharmacology, biochemistry and function. Pharmacol. Therap. 51:13-33.

36. Hsueh, A.J.W. 1995. Designer contraceptive pills. Hum. Reprod. 10: 1997-2000.

37. Pang, D.C. 1992. Tissue and species specificity of cardiac cAMP-phosphodiesterase inhibitors. In Advances in Second Messengers and Phosphoprotein Research. S.J. Strada and H. Hidaka, editors. Raven Press, New York. 307-320. 